\title{
Transcriptomic changes involved in the dedifferentiation of myofibroblasts derived from the lung of a patient with idiopathic pulmonary fibrosis
}

\author{
KENICHI SUZUKI ${ }^{1,2^{*}}$, JUN-DAL KIM $^{3 *}$, KEITA UGAI $^{1,4^{*}}$, SHUICHI MATSUDA $^{1,2^{*}}$, HIDEKI MIKAMI $^{1,2}$, \\ KENTO YOSHIOKA $^{1}$, JUN IKARI ${ }^{2}$, MASAHIKO HATANO ${ }^{1}$, AKIYOSHI FUKAMIZU ${ }^{3}$, \\ KOICHIRO TATSUMI ${ }^{2}$ and YOSHITOSHI KASUYA ${ }^{1,5}$
}

\author{
Departments of ${ }^{1}$ Biomedical Science and ${ }^{2}$ Respirology, Graduate School of Medicine, Chiba University, \\ Chuo-ku, Chiba 260-8670; ${ }^{3}$ Life Science Center for Survival Dynamics, Tsukuba Advanced Research Alliance (TARA), \\ University of Tsukuba, Tsukuba, Ibaraki 305-8577; ${ }^{4}$ Laboratory of Chemical Pharmacology, \\ Graduate School of Pharmaceutical Sciences, Chiba University, Chuo-ku, Chiba 260-8675; \\ ${ }^{5}$ Department of Biochemistry and Molecular Pharmacology, Graduate School of \\ Medicine, Chiba University, Chuo-ku, Chiba 260-8670, Japan
}

Received March 3, 2020; Accepted May 4, 2020

DOI: $10.3892 / \mathrm{mmr} .2020 .11218$

\begin{abstract}
Idiopathic pulmonary fibrosis (IPF) is a fatal lung disease of unknown etiology. Under pathological conditions in lungs with IPF, myofibroblasts serve a key role in fibrogenesis via the accumulation of an excessive amount of extracellular matrix. To develop effective therapeutic interventions against IPF, studies have recently focused on how to dedifferentiate established myofibroblasts. The present study revealed that JQ1, an inhibitor of bromodomain and extra-terminal proteins, markedly suppressed the expression levels of $\alpha$-smooth muscle actin and ED-A-fibronectin in myofibroblasts prepared from the lung of a patient with
\end{abstract}

Correspondence to: Dr Yoshitoshi Kasuya, Department of Biomedical Science, Graduate School of Medicine, Chiba University, 1-8-1 Inohana, Chuo-ku, Chiba 260-8670, Japan

E-mail: kasuya@faculty.chiba-u.jp

${ }^{*}$ Contributed equally

Abbreviations: BET, bromodomain and extra-terminal; BLM, bleomycin; DEGs, differentially expressed genes; ECM, extracellular matrix; FDR, false discovery rate; FMD, fibroblast to myofibroblast differentiation; FN, fibronectin; GO, Gene Ontology; GSEA, gene set enrichment analysis; GSH, glutathione; IPF, idiopathic pulmonary fibrosis; KEGG, Kyoto Encyclopedia of Genes and Genomes; miRNA, microRNA; NES, normalized enrichment score; NHLF, normal human lung fibroblasts; PCA, principal component analysis; SMA, smooth muscle actin; S100A4, S100 calcium-binding protein A4; TGF- $\beta$, transforming growth factor- $\beta$

Key words: idiopathic pulmonary fibrosis, myofibroblast, dedifferentiation, transcriptome analysis, microRNA array end-stage IPF. Furthermore, these findings were supported by transcriptome analysis using RNA sequencing, in which differentially expressed genes (DEGs) downregulated by JQ1 treatment were significantly enriched in the fibrosis-related signaling pathway. On the other hand, the upregulated DEGs in response to JQ1 treatment were significantly enriched in glutathione metabolism, which may affect the cell status of fibroblast/myofibroblast. To the best of our knowledge, this was the first study to comprehensively analyze transcriptome profiles associated with dedifferentiation of IPF myofibroblasts.

\section{Introduction}

Fibrosis is a common pathological outcome of chronic inflammation in various organs. All fibrotic diseases have abnormal and excessive accumulation of extracellular matrix (ECM) components, such as collagen, fibronectin, and elastin in the lesion area, which leads to organ malfunction (1). Idiopathic pulmonary fibrosis (IPF) is a progressive, chronic, and fibrotic interstitial lung disease of unknown etiology and has an extremely poor prognosis in most patients. Nintedanib and pirfenidone have been approved by the Food and Drug Administration as pharmacological therapies for IPF; however, neither of them have been proven effective (2). Therefore, new strategies that can enable the survival of patients suffering from IPF are required.

The activation of ECM-producing myofibroblasts plays a key role in the process of fibrotic tissue remodeling. Fibroblastic foci are formed by myofibroblasts resulting in an aberrant deposition of ECM in the lung. Further, the extent of fibroblastic foci is correlated with the mortality of patients with IPF. Myofibroblasts can differentiate from a variety of precursor cell types, and fibroblast to myofibroblast differentiation (FMD) in response to TGF- $\beta 1$ is well docu- 
mented (3). Therefore, inhibition of FMD may represent an effective therapeutic strategy contributing to the development of fundamental treatments for IPF. However, most patients with IPF showing subjective symptoms are considered under the lung fibrotic stage because of the reduced forced vital capacity (4). This fact tempts us to consider that the resolution of established fibrosis through the promotion of myofibroblast dedifferentiation is a preferable and beneficial intervention for patients with IPF.

Based on this notion, we cultured primary myofibroblasts $(\alpha$-smooth muscle actin (SMA)highED-A-fibronectin (FN)+S100 calcium-binding protein A4 (S100A4)-) derived from a patient with severe lung fibrosis. Furthermore, a small molecule inhibitor library was screened to identify candidate molecules with antifibrotic activity as an index of myofibroblast dedifferentiation defined by the reduced expression of $\alpha$-SMA and ED-A-FN. During screening, a bromodomain and extra-terminal (BET) inhibitor, JQ1, was identified as the most potent dedifferentiating agent for myofibroblasts among several candidates. While JQ1 attenuates pulmonary fibrosis induced by bleomycin (BLM) or radiation, probably through the inhibition of FMD, BET inhibition-induced myofibroblast dedifferentiation and the accompanied gene expression changes remain unknown $(5,6)$. Thus, we performed the transcriptomic analyses of mRNA in patient-derived lung myofibroblasts with or without JQ1 treatment to understand the gene expression modulation.

\section{Materials and methods}

Cell culture. Lung tissue was obtained after surgery from a 46-year-old male patient who underwent lung transplantation due to severe IPF (usual interstitial pneumonia pattern). The patient provided informed consent, and the study was approved by the Ethics Committee of Chiba University, Graduate School of Medicine and Hospital. Lung myofibroblasts were prepared as described in our previous study (7). Briefly, lung tissue was minced into small pieces, and the pieces were incubated in Dulbecco's Modified Eagle Medium (DMEM) supplemented with $1 \mathrm{mg} / \mathrm{ml}$ collagenase type I (Worthington), $0.5 \mathrm{mg} / \mathrm{ml}$ dispase (Thermo Fisher Scientific, Inc.), $2 \mathrm{U} / \mathrm{ml}$ DNase (Qiagen), $0.1 \mathrm{mg} / \mathrm{ml}$ streptomycin, and $100 \mathrm{U} / \mathrm{ml}$ penicillin at $37^{\circ} \mathrm{C}$ for $15 \mathrm{~min}$ with gentle shaking. The pieces were then washed twice with DMEM, transferred to a $90 \mathrm{~mm}$ culture dish (IWAKI Science Products), dipped in culture medium $(10 \%$ fetal bovine serum in DMEM with antibiotics described), and cultured at $37^{\circ} \mathrm{C}$ and $5 \% \mathrm{CO}_{2}$. The outgrown cells were harvested, expanded, and then verified for the population of $\alpha-S M A^{\text {high }} E D-A-F N+S 100 A 4-$ cells as myofibroblasts (Fig. S1). The antibodies used for immunofluorescence staining (for $1 \mathrm{~h}$ at room temperature) were as follows: mouse anti- $\alpha$-SMA antibody (Ab; dilution 1:200; cat. no. ab7817, Sigma-Aldrich), mouse monoclonal anti-FN Ab (clone IST-9, ED-A domain-specific, dilution 1:200; cat. no. sc-59826, Santa Cruz Biotechnology, and rabbit monoclonal anti-S100A4 Ab (dilution 1:250; cat. no. ab124805; Abcam). Actin filaments were detected using Alexa Fluor 488-conjugated phalloidin. Nuclei were stained using (4',6-diamidino-2-phenylindole) DAPI. More than 90\% of the cells were detected as myofibroblasts, named as $46 \mathrm{G}-\mathrm{F}$ cells. As control cells, normal human lung fibroblasts (NHLF) were purchased from Lonza.

Western blotting. After $24 \mathrm{~h}$ of serum deprivation, 46G-F cells or NHLF were treated with JQ1 (1 $\mu \mathrm{M}$, Cayman Chemical), TGF- $\beta 1$ (10 ng/ml, PeproTech) or JQ1 $(1 \mu \mathrm{M})+$ TGF- $\beta 1$ $(10 \mathrm{ng} / \mathrm{ml})$ for an additional $48 \mathrm{~h}$. The cultured cell-derived lysates were subjected to $10 \%$ sodium dodecylsulfate-polyacrylamide gel electrophoresis (SDS-PAGE) and transferred to polyvinylidene difluoride (PVDF) membranes. After washing and blocking, the membranes were incubated with anti- $\alpha$-SMA Ab (dilution 1:1,000), anti-ED-A-FN Ab (dilution $1: 1,000)$, or mouse monoclonal anti- $\beta$-actin Ab (clone AC-15; dilution 1:5,000; cat. no. A5441, Sigma-Aldrich) for $1 \mathrm{~h}$ at room temperature. After washing, the membranes were incubated with appropriate secondary Abs conjugated with horseradish peroxidase for $30 \mathrm{~min}$ at room temperature. The blot was exposed to an X-ray film to visualize the immunoreactive signals by chemiluminescence. The intensity of the signals on the developed X-ray films was measured with ImageJ software (National Institutes of Health).

RNA preparation and reverse transcription-quantitative $P C R$. After $24 \mathrm{~h}$ of serum deprivation, $46 \mathrm{G}-\mathrm{F}$ cells were treated with or without JQ1 $(1 \mu \mathrm{M})$ for a further $48 \mathrm{~h}$ duration. Total RNA of $46 \mathrm{G}-\mathrm{F}$ cells was extracted using ISOGEN plus (Nippon Gene according to the manufacturer's instructions, and the first-strand cDNA was prepared using a RevertAid First Strand cDNA Synthesis Kit (Thermo Fisher Scientific). Thereafter, quantitative PCR (qPCR) was performed using a PowerUp SYBR-Green Master Mix and Pikoreal 96 Real-Time PCR System (Thermo Fisher Scientific, Inc.). Gene expression was normalized to GAPDH mRNA content, and the $\triangle \Delta \mathrm{Cq}$ method was used for all qPCR analyses (Pikoreal software 2.2; Thermo Fisher Scientific, Inc.) (8). The primer sequences used were as follows: ACTA2: (forward) 5'-CTGGCATCGTGCTG GACTCT-3' and(reverse) 5'-GATCTCGGCCAGCCAGATC-3'; FN1: (forward) 5'-GAGCTATTCCCTGCACCTGATG-3' and (reverse) 5'-CGTGCAAGGCAACCACACT-3'; and GAPDH: (forward) 5-GCACCGTCAAGGCTGAGAAC-3' and (reverse) 5'-TGGTGAAGACGCCAGTGGA-3'. Amplification parameters were as follows: 1 cycle of $50^{\circ} \mathrm{C}$ for $2 \mathrm{~min}$ and $95^{\circ} \mathrm{C}$ for $2 \mathrm{~min}$, followed by 40 cycles of $95^{\circ} \mathrm{C}$ for $15 \mathrm{sec}, 55^{\circ} \mathrm{C}$ for $15 \mathrm{sec}$, and $72^{\circ} \mathrm{C}$ for $60 \mathrm{sec}$. The PCR amplifications were performed in triplicates.

RNA sequencing. Total RNA of 46G-F cells with or without JQ1-treatment was extracted using ISOGEN plus. Thereafter, $500 \mathrm{ng}$ of total RNA were ribosomal RNA-depleted using a NEBNext rRNA Depletion Kit (New England Biolabs). The resulting total RNA was converted to an Illumina sequencing library using a NEBNext Ultra Directional RNA Library Prep Kit (New England Biolabs). The library was validated to determine the size distribution and concentration using a bioanalyzer (Agilent Technologies). Sequencing was performed on NextSeq 500 (Illumina) with paired-end 36-base read options. Reads were mapped to the hg19 human reference genome and quantified using CLC Genomics Workbench version 10.1.1 (Qiagen). RNA-seq data sets have been deposited in the Gene Expression Omnibus (GEO) database at the National Center 

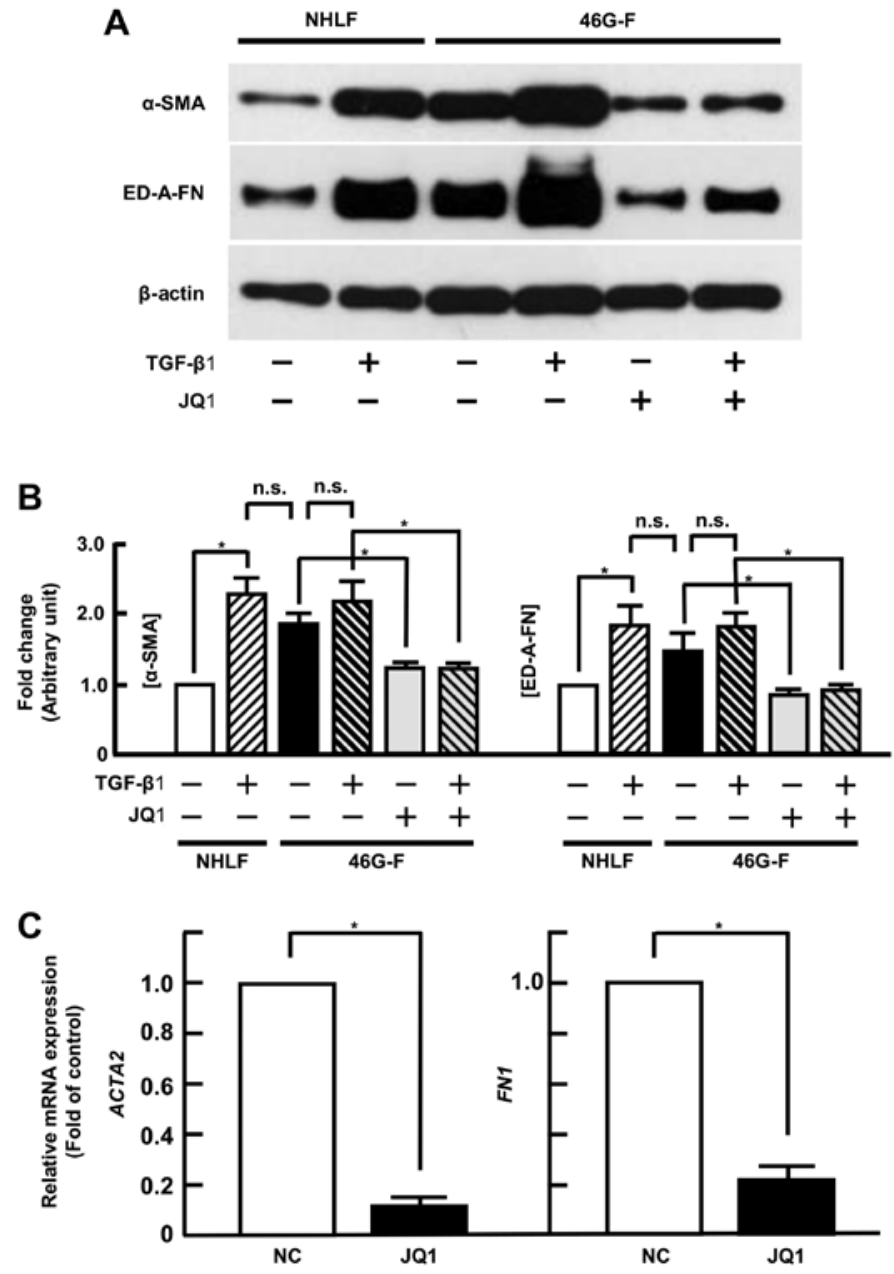

Figure 1. JQ1-induced downregulation of myofibroblast markers. (A) Changes in expression of $\alpha$-SMA and ED-A-FN in NHLF and 46G-F cells observed by western blotting. The signal of $\beta$-actin for each lane was determined as an internal control. (B) The signal of each sample was determined using a densitometer and normalized to each internal control. Signal values are presented as fold changes from the control value of NHLF and as means $\pm \mathrm{SEM}$ ( $\mathrm{n}=3$ ). ${ }^{*} \mathrm{P}<0.05$ (ANOVA followed by Tukey's test). (C) Quantitative PCR analysis of ACTA2 and FN1 expression in 46G-F cells treated with PBS (NC) or JQ1. Quantitative data are presented as fold-changes from the control value and as means \pm SEM ( $n=3)$. * $\mathrm{P}<0.05$ (unpaired Student's t-test). ACTA2, actin $\alpha 2$, smooth muscle; FN1, fibronectin 1; NC, normal control; NHLF, normal human lung fibroblasts; n.s., not significant.

for Biotechnology Information (NCBI) with the accession number GSE140476.

Identification of differentially expressed genes. To analyze the expression pattern of transcripts between the JQ1-treated group and untreated group, read counts were normalized by calculating the number of reads per kilobase per million for each transcript in individual samples using the CLC Genomic Workbench software version 12.0 (9). Filtering characteristics of fold-change -2 to 2 (false discovery rate (FDR) at $\mathrm{P}<0.05)$ were used to identify the DEGs. Subsequently, the distinct gene expression patterns were analyzed comparatively through principal component analysis (PCA) and clustering heatmap. The scatter plot and volcano plot were used to compare the gene expression levels in terms of the $\log _{2}$ fold-change. The gene ontology (GO) (regarding molecular function and biological process) and Kyoto Encyclopedia of Genes and Genomes (KEGG) pathway analyses of differentially expressed genes (DEGs) between the two groups were performed using the ToppGene Suite (https://toppgene.cchmc.org) (10). Gene set enrichment analysis (GSEA) for fibrosis-related pathways derived from the REACTOME (https://reactome.org) pathway analysis was also performed (11).
MicroRNA array analysis. Total RNA of the cells was extracted using ISOGEN plus, and microRNA (miRNA) array analysis was performed by Filgen Co. KEGG pathway analysis of miRNAs was performed using mirPath v.3 software (http://snf-515788.vm.okeanos.grnet.gr). Microarray data sets have been deposited in the NCBI GEO database with the accession number GSE140475.

Statistical analysis. Data are expressed as the mean \pm standard error of the mean (S.E.M). Statistical analysis was conducted using GraphPad Prism Version 6 (GraphPad Software). Statistical significance was determined through an analysis of variance (ANOVA) followed by Tukey's test or unpaired Student's t-test. $\mathrm{P}<0.05$ was considered to indicate a statistically significant difference.

\section{Results}

JQ1-induced dedifferentiation of myofibroblasts from a patient with IPF. FMD is characterized by the upregulated expression of $\alpha$-SMA and ED-A-FN; therefore, these two proteins are recognized as myofibroblast markers $(3,12)$. As shown 
in Fig. 1A, the expression of both $\alpha$-SMA and ED-A-FN in NHLF was observed to be markedly upregulated in response to TGF- $\beta 1$, and the upregulation level was comparable to that of the untreated 46G-F cells. Although stimulation of $46 \mathrm{G}-\mathrm{F}$ cells by TGF- $\beta 1$ tended to upregulate the two markers further, no significant statistical difference was observed between the untreated 46G-F cells and TGF- $\beta 1$-treated 46G-F cells (Fig. 1B), suggesting that the IPF patient-derived myofibroblasts may be well differentiated. In addition to $46 \mathrm{G}-\mathrm{F}$, the differentiation efficiency of two other lines of myofibroblasts from patients with pulmonary fibrosis was also analyzed (data not shown). We used 46G-F cells as the most established myofibroblasts in the present study. JQ1 significantly downregulated the expression of both $\alpha$-SMA and ED-A-FN in 46G-F cells with or without TGF- $\beta 1$, and the level was observed to similar to that of the untreated NHLF (Fig. 1A and B). To elucidate whether the JQ1-induced downregulation of two marker proteins resulted from changes in their mRNA levels in 46G-F cells, qPCR analysis was performed for ACTA2 ( $\alpha$-SMA) and FN1. As shown in Fig. 1C, JQ1 significantly downregulated the expression of ACTA2 and FN1 mRNAs in 46G-F cells. Thus, JQ1 markedly downregulated the expression of the two typical myofibroblast markers at the mRNA and protein levels.

JQ1 broadly suppresses fibrosis-related gene expression. RNA sequencing analysis was performed to understand the molecular basis of functional alteration by JQ1. As shown in Fig. 2A, PCA plots showed two major distinct clusters in which transcripts of samples from JQ1-treated 46G-F cells were clearly separated from those of the normal control group (NC, samples from untreated 46G-F cells). Moreover, the hierarchical clustering analysis showed significant changes in DEGs between the JQ1 and NC groups (adjusted FDR P<0.05). JQ1 downregulated 2,825 DEGs and upregulated 1,330 DEGs compared to the NC group (Fig. 2B and Table SI). The top 10 enriched terms of functional GO annotations and KEGG pathway analysis for the JQ1-downregulated 2,825 DEGs are illustrated in Fig. 2C. Interestingly, these genes were significantly enriched in molecular functions, including collagen binding (GO:0005518), ECM structural constituent conferring tensile strength (GO:0030020), and ECM structural constituent (GO:0005201), and in biological processes, including extracellular structure organization (GO:0043062) and ECM organization (GO:0030198). In accordance with the results of ranked GO terms, KEGG pathway analysis revealed that the DEGs were significantly enriched in the ECM-receptor interaction pathway (BSID:83068). In addition to the downregulation of fibrosis-related gene sets in 46G-F cells, the GO and pathway analysis revealed that JQ1 significantly downregulated molecular functions, including actin binding (GO:0003779) and cytoskeletal protein binding (GO:0008092); biological processes, including cytoskeleton organization (GO:0007010), actin cytoskeleton organization (GO:0030036), and supramolecular fiber organization (GO:0097435); and pathways, including vascular smooth muscle contraction (BSID:96530) and focal adhesion (BSID:83067). To further evaluate the concordant difference of fibrosis-related gene sets between the two groups, GSEA was performed using ranked GO terms in REACTOME pathway analysis. As shown in Fig. 2D, the DEGs were significantly enriched in fibrosis-related gene sets, such as ECM organization, collagen formation, and elastic fiber formation. Moreover, the GSEA graphs and their corresponding normalized enrichment scores (NES) indicated that all three pathways were significantly downregulated in the JQ1 group compared to the NC group. These findings clearly suggest that JQ1 reduces the accumulation of ECM, a main pathological function of myofibroblasts in IPF, wherein JQ1 apparently induces the functional alteration of myofibroblasts. In this alteration process, the marked downregulation of $\alpha$-SMA by JQ1 may play an important role because the actin stress fibers in myofibroblasts possibly affect their morphological features, contraction, and focal adhesion maturation (13).

Profiling of upregulated DEGs in myofibroblasts treated with JQ1. In addition to the downregulated DEGs, profiling of DEGs upregulated by JQ1 in 46G-F cells is of interest to identify the active molecular candidates that regulate the dedifferentiation of myofibroblasts. Scatter plot and volcano plot showed 1,330 DEGs that were significantly upregulated ( $>2$ ) in JQ-1-treated 46G-F cells as compared to NC (Fig. 3A and B). The top 10 enriched terms of functional GO annotations and KEGG pathway analysis for the JQ1-upregulated 1,330 DEGs are illustrated in Fig. 3C. The DEGs were significantly enriched in molecular functions including several transmembrane transporter activities and in biological processes including several metabolic processes, indicating that JQ1-induced dedifferentiation of myofibroblasts is associated with cellular functions. Previous studies have demonstrated a marked decrease in glutathione (L- $\gamma$-glutamyl-L-cysteinyl-glycine, GSH) in the lungs of patients with IPF, and patients with IPF have been treated with $\mathrm{N}$-acetylcysteine (a molecular precursor to $\mathrm{GSH}$ ) monotherapy or combination therapy $(14,15)$. Importantly, the KEGG pathway analysis revealed a significant enrichment of GSH biosynthesis/metabolism pathway-related DEGs. Hierarchical cluster analysis of DEGs involved in the KEGG pathway for GSH metabolism was performed between JQ1 and NC groups, as shown in Fig. 3D. JQ1 significantly upregulated the following genes: microsomal GSH S-transferase 1 (MGST1), GSH S-transferase P1 (GSTP1), GSH S-transferase M3 (GSTM3), glutamate-cysteine ligase modifier subunit (GCLM), ornithine decarboxylase 1 (ODC1), glucose-6-phosphate dehydrogenase $(G 6 P D)$, phosphogluconate dehydrogenase $(P G D), \mathrm{GSH}$ synthetase $(G S S), \mathrm{GSH}$ peroxidase 3 (GPX3), and GSH-disulfide reductase (GSR).

\section{Discussion}

Myofibroblasts have been considered to be a terminally differentiated fibrotic cell phenotype (16). However, it has been recently demonstrated that myofibroblasts can dedifferentiate (17). When the patient is diagnosed with IPF, activated myofibroblasts may already harbor the fibroblastic foci (4). To discover the potential therapeutic agents for IPF, an assay system with myofibroblast dedifferentiation considered as an index may be useful (18). In addition, the utilization of IPF myofibroblasts in the assay is preferable. The prostaglandin E2 capable of dedifferentiating TGF- $\beta 1$-established myofibroblasts failed to affect the IPF myofibroblasts, suggesting that the in vitro FMD induced by TGF- $\beta 1$ may not replicate genetic and epigenetic 
A

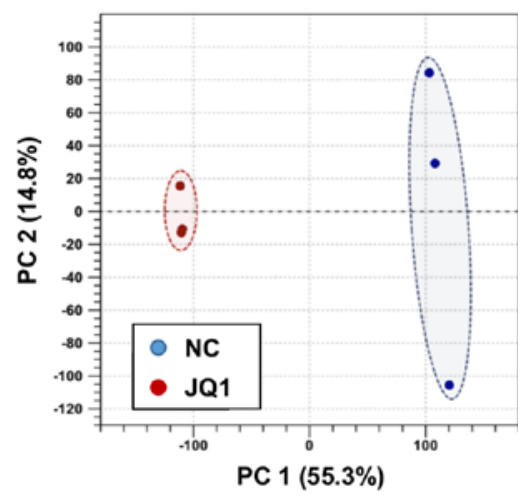

B

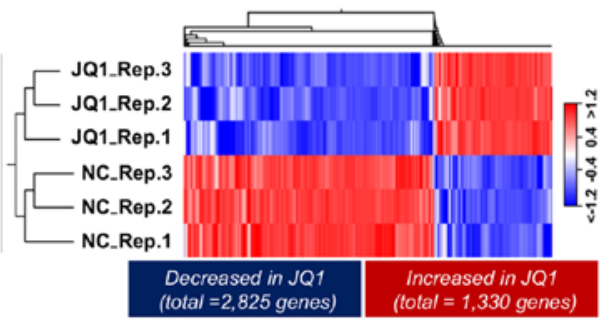

C

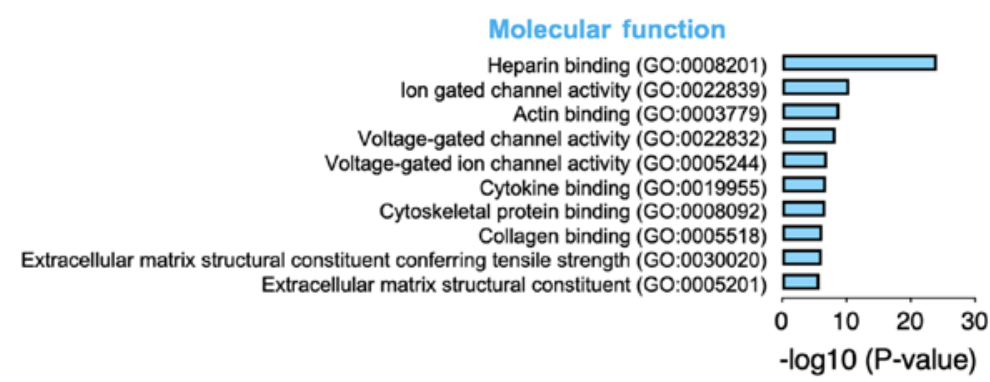

Biological process

Negative regulation of multicellular organismal process (GO:0051241) Skeletal system development (GO:0001501) Cytoskeleton organization (GO:0007010) Actin cytoskeleton organization (GO:0030036) Extracellular structure organization (GO:0043062) Supramolecular fiber organization (GO:0097435 Extracellular matrix organization (GO:0030198) Actin filament-based process (GO:0030029) Biological adhesion (GO:0022610) Cell adhesion (GO:0007155)

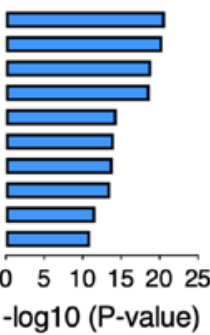

KEGG pathway

Amyotrophic lateral sclerosis (ALS) (BSID:83099) Vascular smooth muscle contraction (BSID:96530) Calcium signaling pathway (BSID:83050) TNF signaling pathway (BSID:812256) NOD-like receptor signaling pathway (BSID:122191) p53 signaling pathway (BSID:83055) Focal adhesion (BSID:83067) AGE-RAGE signaling pathway in diabetic complications (BSID:1319988) ECM-receptor interaction (BSID:83068) Protein digestion and absorption (BSID:172847)

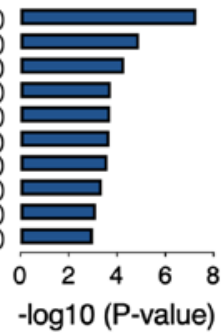

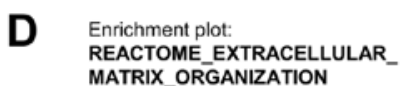

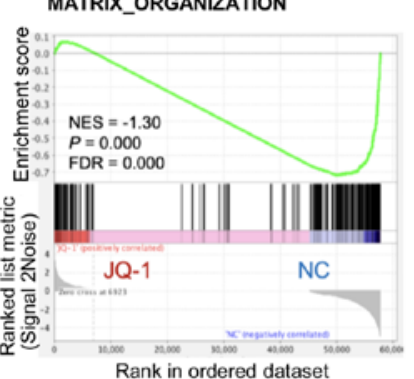

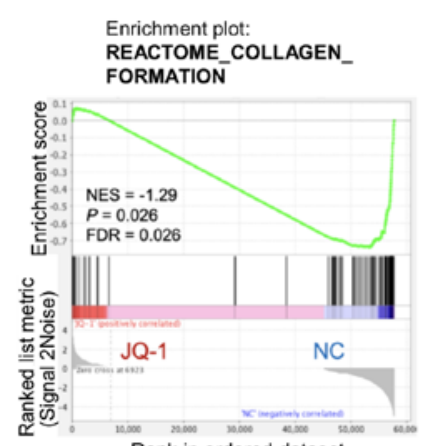

Rank in ordered dataset
Enrichment plot: REACTOME_ELASTIC_FIBER FORMATION

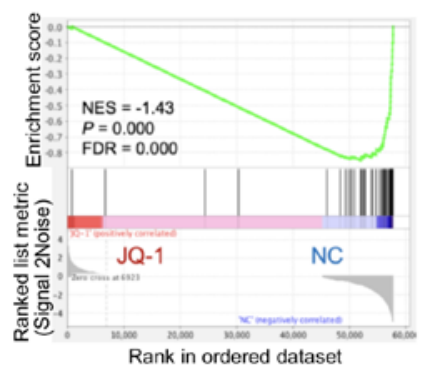

Enrichment profile - Hits Ranking metric scores

Figure 2. Identification of DEGs between NC and JQ-1 treated cells. (A) PC analysis. PC1 (x-axis, 55.3\%) and PC2 (y-axis, $14.8 \%$ ) of the total variation in expressed genes of all cells. (B) Hierarchical clustering of the expression profiles of DEGs between NC and JQ-1-treated groups (adjusted FDR P<0.05). Red represents high relative expression, and blue represents low relative expression. Upregulated DEGs (1,330 genes; JQ-1 vs. NC) and downregulated DEGs (2,825 genes; JQ-1 vs. NC). (C) GO and pathway analysis of downregulated DEGs. Top 10 enriched GO terms associated with molecular functions (top) and biological processes (middle), and KEGG pathway analysis (bottom). (D) Gene set enrichment analysis plots show enrichment of fibrosis-related gene sets (extracellular matrix organization, collagen formation and elastic fiber formation) in two groups. The NES, normal P-value and FDR q value are also indicated. DEGs, differentially expressed genes; FDR, false discovery rate; GO, Gene Ontology; KEGG, Kyoto Encyclopedia of Genes and Genomes; NC, normal control; NES, normalized enrichment score; PC, principal component. 


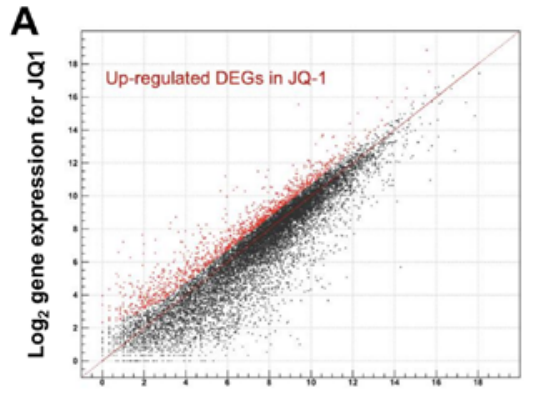

$\log _{2}$ gene expression for NC
B

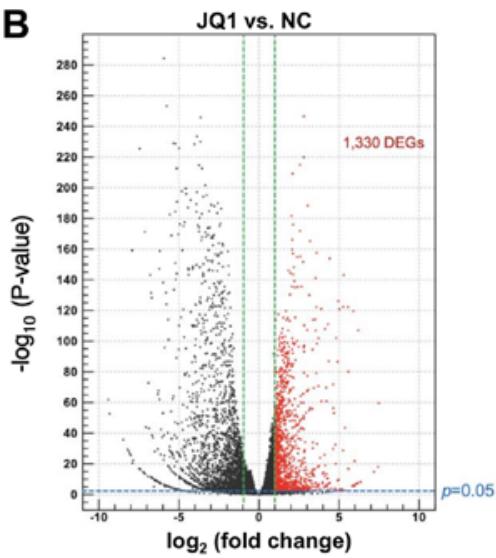

\section{C}

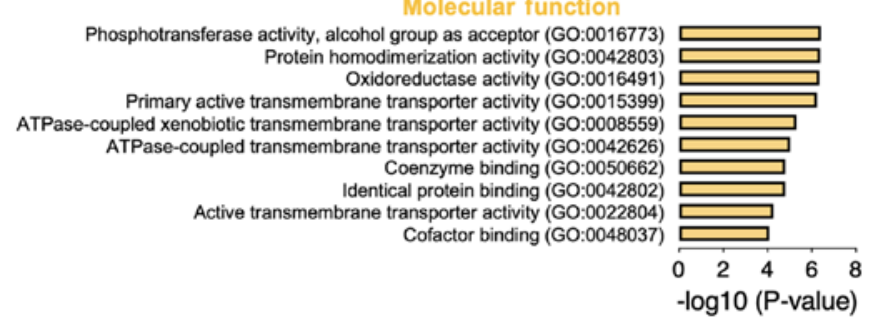

Biological process Response to oxygen-containing compound (GO:1901700)
Central nervous system development (GO:0007417) Central nervous system development (GO:0007417)
Response to peptide (GO:1901652) Cellular chemical homeostasis (GO:0055082) Cell-cell signaling (GO:0007267) Chemical homeostasis (GO:0048878) Chemical homeostasis (GO:0048878)
Cellular homeostasis (GO:0019725) Cellular homeostasis (GO:0019725)
Carboxylic acid metabolic process (GO:0019752) Organic acid metabolic process (GO:0006082)
Oras Oxoacid metabolic process (GO:0043436) $\begin{array}{llll}0 & 5 & 10 & 15\end{array}$ -log10 (P-value)

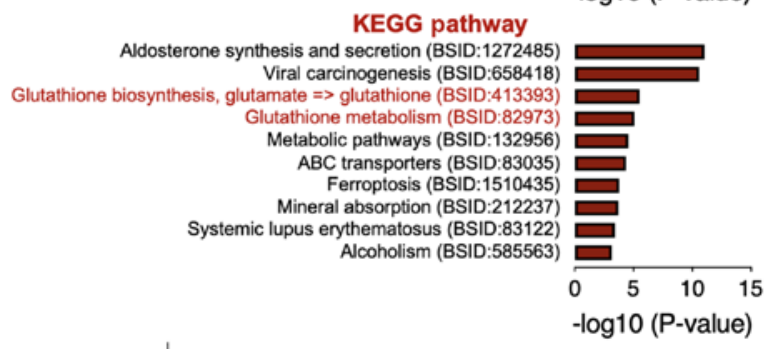

D

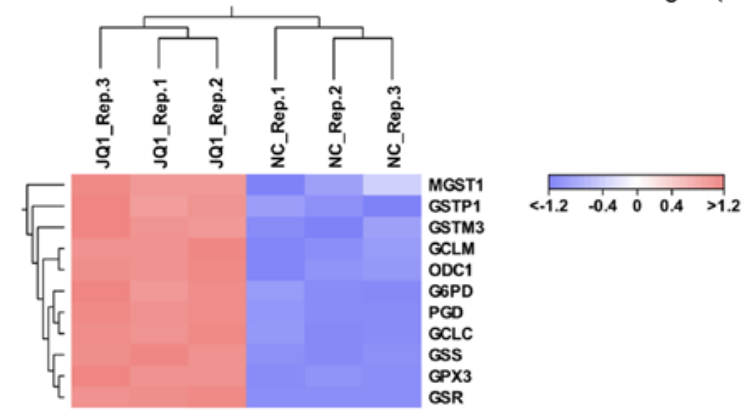

Figure 3. Identification and enrichment analysis of upregulated DEGs in JQ-1-treated cells. (A) Scatter plot and (B) volcano plot of expression profiles between two groups. Points colored in red were significantly different (adjusted false discovery rate $\mathrm{P}<0.05$ ). (C) Functional enrichment analysis of 1,330 upregulated DEGs. Top 10 enriched Gene Ontology terms associated with molecular functions and biological processes, and KEGG pathways, and the negative $\log _{10}$ of the P-value. (D) Hierarchical cluster analysis of DEGs in the KEGG pathway for glutathione metabolism between two groups. Red represents high relative expression and blue represents low relative expression. DEGs, differentially expressed genes; KEGG, Kyoto Encyclopedia of Genes and Genomes; NC, normal control.

changes in IPF myofibroblasts $(19,20)$. Therefore, in the present study, we prepared myofibroblasts established from a patient with end-stage IPF/usual interstitial pneumonia (Fig. S1).

During the screening of a small library of epigenetics-related inhibitors (80 compounds, Sigma-Aldrich), JQ1 showed the highest ability to dedifferentiate IPF myofibroblasts among several candidates (data not shown). It has been clearly demonstrated that JQ1 can ameliorate lung fibrosis induced by BLM and radiation in animal models, probably through TGF- $\beta 1$-mediated FMD $(5,6)$. However, the involved molecular 
mechanisms appear to be different between the inhibition of TGF- $\beta 1$-mediated FMD by JQ1 and the reversal of established myofibroblasts by JQ1. To date, the transcriptional profiles related to the dedifferentiation of IPF myofibroblasts remain unknown. Hence, we have investigated the DEGs involved in the dedifferentiation of IPF myofibroblasts treated with JQ1, a potent reversal agent. The functional GO annotations and KEGG pathway analysis of the genes downregulated by the JQ1-treatment clearly demonstrated the enrichment of ECM-related gene sets (Fig. 3C). Furthermore, another pathway analysis, REACTOME, followed by GSEA also showed that fibrosis-related gene sets could be enriched in the downregulated genes (Fig. 3D). Moreover, the changes in cytoskeletal organization and contractile activity were ranked in the top 10 of functional GO annotations and KEGG pathway analysis, which were probably associated with the downregulation of $\alpha$-SMA. Collectively, the results imply that JQ1 can reverse IPF myofibroblasts and suppress their main pathological aspects.

Interestingly, KEGG pathway analysis of the upregulated gene sets by JQ1 revealed that the GSH biosynthesis/metabolism pathway could be potentiated by the reversal of IPF myofibroblasts (Fig. 3C). This notion was supported by the hierarchical cluster analysis of GSH metabolism-related genes. Intracellular GSH has been reported to evoke an antioxidant function, and pretreatment of fibroblasts with a precursor of GSH, $\gamma$-glutamylcysteine ethyl ester, was reported to ameliorate TGF- $\beta 1$-induced FMD $(21,22)$. In contrast, inhibition of the GSTP1 gene-encoding GST-Pi1, which catalyzes FAS S-glutathionylation by utilizing GSH, has been reported to ameliorate established lung fibrosis (23). Therefore, GSH and GSH-related enzyme-mediated intracellular balance of oxidation/reduction states may correlate with the cell status of fibroblast/myofibroblast. Ferroptosis observed in the top 10 of the KEGG pathway analysis was also notable. To elucidate whether the ferroptotic pathway is involved in the dedifferentiation process of IPF myofibroblasts, we investigated the effect of ferrostatin-1, an inhibitor of ferroptosis, on the downregulation of $\alpha$-SMA and ED-A-FN by JQ-1. However, ferrostatin-1 did not show any effect on JQ1 action, suggesting that the ferroptotic pathway may not function in the dedifferentiation process (data not shown). Myofibroblasts are resistant to FasL/FAS axis-mediated apoptosis compared to the normal fibroblasts (24). Hence, dedifferentiated myofibroblasts might acquire high sensitivity to apoptosis as well as ferroptosis. Ferroptosis is regulated by glutathione and iron metabolism-dependent lipid peroxidation (25). Therefore, the correlation between the two picked-up pathways is of interest in estimating the cell status.

The BET proteins function as epigenetic reader proteins that specifically recognize acetyl-lysine residues located on histones and promote the recruitment of transcription factors and chromatin organizers. Among the BET family proteins, the involvement of BRD4 in the progression of fibrotic disease in several organs, including IPF, has been well characterized (26). Epigenetics involved in the pathogenesis of IPF has been thoroughly investigated in various studies. The studies have revealed that a DNA hypermethylation-associated decrease in mRNA expression can be detected in 16 genes of patients with IPF (27), histone deacetylase blockers efficiently inhibit FMD and the proliferation of IPF fibroblasts (28), and miRNAs function as profibrotic and antifibrotic mediators in IPF (29). We then performed miRNA microarray analysis using total RNA isolated from 46G-F cells treated with or without JQ1 (Tables SII-SIV; NCBI GEO accession number GSE140475). A previous report by Mullenbrock et al (30) performed comprehensive analysis of miRNAs differentially expressed in fibroblasts from the lungs of patients with IPF and control groups. Therefore, information regarding upregulated or downregulated miRNAs (three upregulated miRNAs and 16 downregulated miRNAs) in IPF fibroblasts compared to control fibroblasts was obtained from a previous study and checked against the data of the present study to evaluate the association of the overlapped miRNAs with lung fibrosis. As shown in Fig. S2, the Venn diagram revealed that our upregulated miRNAs overlapped six of the 16 downregulated miRNAs (hsa-miR-17-5p, hsa-miR-21-3p, hsa-miR-20a-5p, hsa-miR-93-5p, hsa-miR-155-5p, and hsa-miR-345-5p). Among these six differentially expressed miRNAs, both miR-17-5p and miR-20a-5p are the members of the miR-17-92 cluster, which can reverse IPF fibroblast activation, but are downregulated in fibroblastic foci in the lung with IPF (31). Moreover, miR-93-5p can function as a negative regulator of TGF- $\beta$ signaling but is downregulated in the lungs of slowly progressive IPF $(32,33)$. These antifibrotic miRNAs were observed to be upregulated in the JQ1-treated 46G-F cells in which the fibrosis-related genes were downregulated (Fig. 2). Hence, provided that JQ1 could reverse the characteristics of IPF myofibroblasts, the antifibrotic miRNAs downregulated in IPF fibroblasts may be recovered by treating 46G-F cells with JQ1. In contrast, miR-21 evokes fibrogenic action and is predominantly upregulated in myofibroblasts in the lungs of mice instilled with BLM as well as patients with IPF (34). Likewise, the expression level of miR-155 in peripheral blood mononuclear cells is correlated with progressive systemic sclerosis interstitial lung disease (35). Whether these profibrotic miRNAs are upregulated by the compensatory actions of JQ1 remains unknown but is of particular interest. Additionally, the downregulated miRNAs identified in the present study overlapped three of the 16 downregulated miRNAs (hsa-miR-99a-5p, hsa-miR-146b-5p, and hsa-miR-125b-2-3p). The mechanism regarding the relationship of these three miRNAs with the pathogenesis of IPF remains unclear, and further studies are required. KEGG pathway analysis for each differentially expressed miRNA is shown in Fig. S2B. Paying attention to the top 5 target pathways of each miRNA from the viewpoint of the beneficial effects of JQ1, cancer-related pathways covered four different miRNAs. This result is expected since role of BET proteins as promising therapeutic targets in cancer is widely accepted (36).

In conclusion, we have clearly demonstrated that the inhibition of BET proteins by JQ1 could dedifferentiate IPF myofibroblasts. Furthermore, our study has provided new perspectives on gene and miRNA expression profiles associated with the reversal of IPF lung-derived myofibroblasts. This notion will be more convincing if several established IPF myofibroblasts are used in future studies, although the myofibroblasts derived from an end-stage IPF patient were used in the present study. 


\section{Acknowledgements}

Not applicable.

\section{Funding}

This work was supported by a Therapeutics Research Initiative Grant from Chiba University, School of Medicine (grant nos. 2018-G7 and 2019-Y10) and the Cooperative Research Project Program of Life Science Center for Survival Dynamics, Tsukuba Advanced Research Alliance (TARA Center), University of Tsukuba, Japan (grant no. 201905).

\section{Availability of data and materials}

The datasets used and/or analyzed during the current study are available from the corresponding author on reasonable request.

\section{Authors' contributions}

JDK, MH, AF, KT and YK developed the concept and designed the experiments. KS, JDK, KU, SM, HM, KY, JI and YK performed the experiments. JDK, SM and YK wrote the paper. All authors discussed the results and implications, and commented on the manuscript at all stages. All authors read and approved the final manuscript.

\section{Ethics approval and consent to participate}

The patient involved in the present study provided written informed consent, and the study was approved by the Ethics Committee of Chiba University, Graduate School of Medicine and Hospital.

\section{Patient consent for publication}

Not applicable.

\section{Competing interests}

The authors declare that they have no competing interests.

\section{References}

1. Wynn TA and Ramalingam TR: Mechanisms of fibrosis: therapeutic translation for fibrotic disease. Nat Med 18: 1028-1040, 2012.

2. Lederer DJ and Martinez FJ: Idiopathic Pulmonary Fibrosis. N Engl J Med 378: 1811-1823, 2018.

3. Kendall RT and Feghali-Bostwick CA: Fibroblasts in fibrosis: Novel roles and mediators. Front Pharmacol 5: 123, 2014.

4. King TE Jr, Schwarz MI, Brown K, Tooze JA, Colby TV, Waldron JA Jr, Flint A, Thurlbeck W and Cherniack RM: Idiopathic pulmonary fibrosis: relationship between histopathologic features and mortality. Am J Respir Crit Care Med 164 1025-1032, 2001.

5. Tang X,Peng R,Phillips JE,Deguzman J, Ren Y,Apparsundaram S, Luo Q, Bauer CM, Fuentes ME, DeMartino JA, et al: Assessment of Brd4 inhibition in idiopathic pulmonary fibrosis lung fibroblasts and in vivo models of lung fibrosis. Am J Pathol 183: 470-479, 2013

6. Wang J, Zhou F, Li Z, Mei H, Wang Y, Ma H, Shi L, Huang A, Zhang T, Lin Z, et al: Pharmacological targeting of BET proteins attenuates radiation-induced lung fibrosis. Sci Rep 8: 998, 2018.
7. Yamazaki R, Kasuya Y, Fujita T, Umezawa H, Yanagihara M, Nakamura H, Yoshino I, Tatsumi K and Murayama T: Antifibrotic effects of cyclosporine A on TGF-beta1-treated lung fibroblasts and lungs from bleomycin-treated mice: role of hypoxia-inducible factor-1alpha. FASEB J 31: 3359-3371, 2017.

8. Livak KJ and Schmittgen TD: Analysis of relative gene expression data using real-time quantitative PCR and the $2^{-\Delta \Delta C q}$ method. Methods 25: 402-408, 2001.

9. Ohkuro M, Kim JD, Kuboi Y, Hayashi Y, Mizukami H, Kobayashi-Kuramochi H, Muramoto K, Shirato M, Michikawa-Tanaka F, Moriya J, et al: Calreticulin and integrin alpha dissociation induces anti-inflammatory programming in animal models of inflammatory bowel disease. Nat Commun 9: 1982, 2018.

10. Chen J, Bardes EE, Aronow BJ and Jegga AG: ToppGene Suite for gene list enrichment analysis and candidate gene prioritization. Nucleic Acids Res 37: W305-W311, 2009.

11. Subramanian A, Tamayo P, Mootha VK, Mukherjee S, Ebert BL, Gillette MA,Paulovich A,Pomeroy SL,Golub TR,LanderES, etal: Gene set enrichment analysis: a knowledge-based approach for interpreting genome-wide expression profiles. Proc Natl Acad Sci U S A 102: 15545-15550, 2005.

12. Serini G, Bochaton-Piallat ML, Ropraz P, Geinoz A, Borsi L, Zardi L and Gabbiani G: The fibronectin domain ED-A is crucial for myofibroblastic phenotype induction by transforming growth factor-beta1. J Cell Biol 142: 873-881, 1998.

13. Sandbo N and Dulin N: Actin cytoskeleton in myofibroblast differentiation: ultrastructure defining form and driving function. Transl Res 158: 181-196, 2011.

14. Hunninghake GW: Antioxidant therapy for idiopathic pulmonary fibrosis. N Engl J Med 353: 2285-2287, 2005.

15. Raghu G, Noth I and Martinez F: N-acetylcysteine for idiopathic pulmonary fibrosis: the door is still open. Lancet Respir Med 5: e1-e2, 2017.

16. Pierce EM, Carpenter K, Jakubzick C, Kunkel SL, Flaherty KR, Martinez FJ and Hogaboam CM: Therapeutic targeting of CC ligand 21 or $\mathrm{CC}$ chemokine receptor 7 abrogates pulmonary fibrosis induced by the adoptive transfer of human pulmonary fibroblasts to immunodeficient mice. Am J Pathol 170: 1152-1164, 2007.

17. Yang $X$, Chen B, Liu T and Chen X: Reversal of myofibroblast differentiation: a review. Eur J Pharmacol 734: 83-90, 2014.

18. Sieber P, Schäfer A, Lieberherr R, Le Goff F, Stritt M, Welford WD, Gatfield J, Peter O, Nayler O and Lüthi U: Novel high-throughput myofibroblast assays identify agonists with therapeutic potential in pulmonary fibrosis that act via EP2 and EP4 receptors. PLoS One 13: e0207872, 2018.

19. Garrison G, Huang SK, Okunishi K, Scott JP, Kumar Penke LR, Scruggs AM and Peters-Golden M: Reversal of myofibroblast differentiation by prostaglandin E(2). Am J Respir Cell Mol Biol 48: 550-558, 2013.

20. Huang SK, Fisher AS, Scruggs AM, White ES, Hogaboam CM, Richardson BC and Peters-Golden M: Hypermethylation of PTGER2 confers prostaglandin E2 resistance in fibrotic fibroblasts from humans and mice. Am J Pathol 177: 2245-2255, 2010.

21. Anathy V, Roberson EC, Guala AS, Godburn KE, Budd RC and Janssen-Heininger YM: Redox-based regulation of apoptosis: S-glutathionylation as a regulatory mechanism to control cell death. Antioxid Redox Signal 16: 496-505, 2012.

22. Ono A, Utsugi M, Masubuchi K, Ishizuka T, Kawata T, Shimizu Y, Hisada T, Hamuro J, Mori M and Dobashi K: Glutathione redox regulates TGF-beta-induced fibrogenic effects through Smad3 activation. FEBS Lett 583: 357-362, 2009.

23. McMillan DH, van der Velden JL, Lahue KG, Qian X, Schneider RW, Iberg MS, Nolin JD, Abdalla S, Casey DT, Tew KD, et al: Attenuation of lung fibrosis in mice with a clinically relevant inhibitor of glutathione-S-transferase $\pi$. JCI Insight 1: e85717, 2016.

24. Wynes MW, Edelman BL, Kostyk AG, Edwards MG, Coldren C, Groshong SD, Cosgrove GP, Redente EF, Bamberg A, Brown KK, et al: Increased cell surface Fas expression is necessary and sufficient to sensitize lung fibroblasts to Fas ligation-induced apoptosis: implications for fibroblast accumulation in idiopathic pulmonary fibrosis. J Immunol 187: 527-537, 2011.

25. Gong Y, Wang N, Liu N and Dong H: Lipid peroxidation and GPX4 inhibition are common causes for myofibroblast differentiation and ferroptosis. DNA Cell Biol 38: 725-733, 2019.

26. Stratton MS, Haldar SM and McKinsey TA: BRD4 inhibition for the treatment of pathological organ fibrosis. F1000Res 6: 1015, 2017. 
27. Sanders YY, Ambalavanan N, Halloran B, Zhang X, Liu H, Crossman DK, Bray M, Zhang K, Thannickal VJ and Hagood JS: Altered DNA methylation profile in idiopathic pulmonary fibrosis. Am J Respir Crit Care Med 186: 525-535, 2012.

28. Davies ER, Haitchi HM, Thatcher TH, Sime PJ, Kottmann RM, Ganesan A, Packham G, O'Reilly KM and Davies DE: Spiruchostatin A inhibits proliferation and differentiation of fibroblasts from patients with pulmonary fibrosis. Am J Respir Cell Mol Biol 46: 687-694, 2012.

29. Tzouvelekis A and Kaminski N: Epigenetics in idiopathic pulmonary fibrosis. Biochem Cell Biol 93: 159-170, 2015.

30. Mullenbrock S, Liu F, Szak S, Hronowski X, Gao B, Juhasz P, Sun C, Liu M, McLaughlin H, Xiao Q, et al: Systems analysis of transcriptomic and proteomic profiles identifies novel regulation of fibrotic programs by miRNAs in pulmonary fibrosis fibroblasts. Genes (Basel) 9: E588, 2018.

31. DakhlallahD,BatteK,Wang Y,Cantemir-StoneCZ, YanP,NuovoG, Mikhail A, Hitchcock CL, Wright VP, Nana-Sinkam SP, et al: Epigenetic regulation of miR-17-92 contributes to the pathogenesis of pulmonary fibrosis. Am J Respir Crit Care Med 187: 397-405, 2013

32. Lyu X, Fang W, Cai L, Zheng H, Ye Y, Zhang L, Li J, Peng H, Cho WC, Wang E, et al: TGF $\beta \mathrm{R} 2$ is a major target of miR-93 in nasopharyngeal carcinoma aggressiveness. Mol Cancer 13: 51, 2014.
33. Oak SR, Murray L, Herath A, Sleeman M, Anderson I, Joshi AD, Coelho AL, Flaherty KR, Toews GB, Knight D, et al: A micro RNA processing defect in rapidly progressing idiopathic pulmonary fibrosis. PLoS One 6: e21253, 2011.

34. Liu G, Friggeri A, Yang Y, Milosevic J, Ding Q, Thannickal VJ, Kaminski N and Abraham E: miR-21 mediates fibrogenic activation of pulmonary fibroblasts and lung fibrosis. J Exp Med 207: 1589-1597, 2010.

35. Christmann RB, Wooten A, Sampaio-Barros P, Borges CL, Carvalho CR, Kairalla RA, Feghali-Bostwick C, Ziemek J, Mei Y, Goummih S, et al: miR-155 in the progression of lung fibrosis in systemic sclerosis. Arthritis Res Ther 18: 155, 2016.

36. Fu LL, Tian M, Li X, Li JJ, Huang J, Ouyang L, Zhang Y and Liu B: Inhibition of BET bromodomains as a therapeutic strategy for cancer drug discovery. Oncotarget 6: 5501-5516, 2015.

This work is licensed under a Creative Commons Attribution-NonCommercial-NoDerivatives 4.0 International (CC BY-NC-ND 4.0) License. 